\title{
Multiconfiguration time-dependent Hartree impurity solver for nonequilibrium dynamical mean-field theory
}

\author{
Karsten Balzer, ${ }^{1,2, *}$ Zheng Li, ${ }^{2,3}$ Oriol Vendrell, ${ }^{2, \dagger}$ and Martin Eckstein ${ }^{1,2}$ \\ ${ }^{1}$ Max Planck Research Department for Structural Dynamics, University of Hamburg, 2607 Hamburg, Germany \\ ${ }^{2}$ Center for Free-Electron Laser Science, DESY, Notkestraße 85, 22607 Hamburg, Germany \\ ${ }^{3}$ Department of Physics, University of Hamburg, 20355 Hamburg, Germany
}

(Received 23 July 2014; revised manuscript received 23 December 2014; published 26 January 2015)

\begin{abstract}
Nonequilibrium dynamical mean-field theory (DMFT) solves correlated lattice models by obtaining their local correlation functions from an effective model consisting of a single impurity in a self-consistently determined bath. The recently developed mapping of this impurity problem from the Keldysh time contour onto a time-dependent single-impurity Anderson model (SIAM) [C. Gramsch et al., Phys. Rev. B 88, 235106 (2013)] allows one to use wave-function-based methods in the context of nonequilibrium DMFT. Within this mapping, long times in the DMFT simulation become accessible by an increasing number of bath orbitals, which requires efficient representations of the time-dependent SIAM wave function. These can be achieved by the multiconfiguration time-dependent Hartree (MCTDH) method and its multilayer extensions. We find that MCTDH outperforms exact diagonalization for large baths in which the latter approach is still within reach and allows for the calculation of SIAMs beyond the system size accessible by exact diagonalization. Moreover, we illustrate the computation of the self-consistent two-time impurity Green's function within the MCTDH second quantization representation.
\end{abstract}

DOI: 10.1103/PhysRevB.91.045136

\section{INTRODUCTION}

Pump-probe experiments with femtosecond time resolution can access the real-time dynamics in materials with strong correlation effects on the time scale of the electronic motion $[1,2]$ and reveal striking phenomena such as photoinduced insulator-to-metal transitions in correlated Mott and chargetransfer insulators [3,4] and pump-induced melting and recovery of charge density waves [5]. In order to understand the underlying physical scenario revealed by those experiments, growing theoretical effort has been devoted to establishing a microscopic description of strongly correlated lattice models out of equilibrium. Yet the numerical simulation of nonequilibrium quantum many-body systems beyond weak-coupling perturbation theory remains a challenge, in particular, for extended systems in dimensions greater than one, where the time-dependent density matrix renormalization-group method [6] or an exact solution of the Schrödinger equation is no longer feasible.

A promising framework to capture both ultrafast dynamics and strong electronic correlations is the nonequilibrium formulation of dynamical mean-field theory (DMFT) [7-9], which generalizes DMFT [10] to the Keldysh formalism. In the framework of DMFT, a lattice model such as the Hubbard model is mapped onto an effective impurity model, which consists of a single site of the lattice (impurity) coupled to a noninteracting medium, where electrons are exchanged between the impurity site and the medium. One of the key developments for advancing DMFT to the nonequilibrium regime is to establish methods to solve the real-time dynamics of this impurity model far from equilibrium. Impurity solvers that have been used so far include real-time continuous-time quantum Monte Carlo [11], which is numerically exact but

\footnotetext{
*karsten.balzer@mpsd.cfel.de

†oriol.vendrell@cfel.de
}

PACS number(s): 71.27.+a, 71.10.Fd, 05.70.Ln

restricted to short times, as well as strong-coupling [12] and weak-coupling [13] expansions, which have been employed in many studies (see Ref. [9] for an overview) but are restricted to certain parameter regimes. Recently, a Hamiltonian-based impurity solver scheme has been developed, which further maps the DMFT impurity model onto a single-impurity Anderson model (SIAM) with a finite number of bath orbitals [14]. The latter is then solved to self-consistency by an exact diagonalization (ED) method being equivalent to the time-dependent full configuration interaction [15], and one is thus not restricted to either weak or strong on-site Coulomb interactions.

The mapping of the DMFT impurity model to a SIAM is similar to the related ED approach to DMFT in equilibrium [10], but nevertheless there are important conceptual differences: Apart from the description of the initial state, the representation of the DMFT bath can be made exact for small times, while it requires an increasing number of bath orbitals to reach longer times [14,16] (note that other representation schemes might be useful to obtain qualitatively correct descriptions with few bath orbitals [17] or to describe the steady state [18]). Intuitively, increasing the number of bath orbitals allows the discrete model to develop the finite memory time that is inherent in the original infinite DMFT bath; i.e., the state can explore a larger Hilbert space without ever returning close to its initial state. Despite the accuracy of the ED method, the exponential scaling of the Hilbert-space dimension as a function of the number of bath orbitals therefore prohibits us from acquiring the dynamics at long time scales.

Various approaches in different areas of physics have been developed to overcome this course of dimensionality by finding efficient representations of the wave function. In condensed matter physics, this includes the (time-dependent) density matrix renormalization group [6], which is based on a matrix product state representation and tensor-network representations of many-fermion states [19-21]. In the present work, we introduce the multiconfiguration time-dependent Hartree (MCTDH) method, which was originally developed for the 
time propagation of nuclear wave packets in molecular quantum dynamics [22,23], to treat the real-time dynamics of the SIAM. The MCTDH method provides a route to represent the wave function with a minimal set of time-dependent basis functions that comove with the evolving state. This feature can lead to a tremendous reduction in the configuration space. Moreover, the more powerful extension of the MCTDH method, the multilayer multiconfiguration time-dependent Hartree (MLMCTDH) method [24-26], allows for well-adapted tree-tensor network decompositions of the many-body wave function.

While MCTDH propagation schemes have recently been used to study transport in the Anderson and Anderson-Holstein model [27,28], the requirements for a nonequilibrium DMFT calculation are often quite demanding: The Hamiltonian representation of the DMFT impurity model typically implies strongly time-dependent parameters, the regime of interest includes strong Coulomb interactions, and, in particular, a fast calculation is required because one needs to perform a large number of simulations to obtain the impurity Green's function as a function of two time variables. In order to judge the usefulness of the MCTDH method for nonequilibrium DMFT it is thus important to provide a comparison of the numerical performance of the method, i.e., to analyze the ability of the ansatz to compress the SIAM wave function in the typical parameter regime relevant for DMFT and thus to improve on the exponential increase in the numerical effort on the simulation time which we have described above. This is one main goal of this paper.

The article is organized as follows. In Sec. II, we give a brief overview of the nonequilibrium DMFT and outline the mapping to a SIAM underlying the Hamiltonian-based impurity solver. We then introduce the MCTDH method in Sec. II B and discuss its implementation using the Fock-space formalism in Sec. II C. Thereafter, in Sec. III, we present numerical results to assess the performance of the MCTDH method as an impurity solver in the context of DMFT. Finally, Sec. IV provides a general conclusion.

\section{THEORETICAL FRAMEWORK}

In order to combine the nonequilibrium DMFT and the MCTDH method, which we propose as impurity solver, we first give a brief introduction to the DMFT framework. For a comprehensive introduction to nonequilibrium DMFT and its applications the reader is referred to Ref. [9].

From a general perspective, we are interested in the realtime evolution of a lattice quantum many-body system like the single-band Hubbard model,

$$
H(t)=\sum_{i j \sigma} t_{i j}(t) c_{i \sigma}^{\dagger} c_{j \sigma}+U(t) \sum_{i}\left(n_{i \uparrow}-\frac{1}{2}\right)\left(n_{i \downarrow}-\frac{1}{2}\right),
$$

which is initially in thermodynamic equilibrium at temperature $T=1 / \beta$ and evolves unitarily under the time-dependent Hamiltonian $H(t)$. In Eq. (1), the operator $c_{i \sigma}^{\dagger}\left(c_{i \sigma}\right)$ creates (annihilates) an electron with spin $\sigma$ on site $i$ of the crystal lattice, $n_{i \sigma}$ is the spin-resolved density, $t_{i j}(t)$ is the hopping matrix element between site $i$ and site $j$, and $U(t)$ denotes the local Coulomb repulsion. Below, we adopt a parametrized model
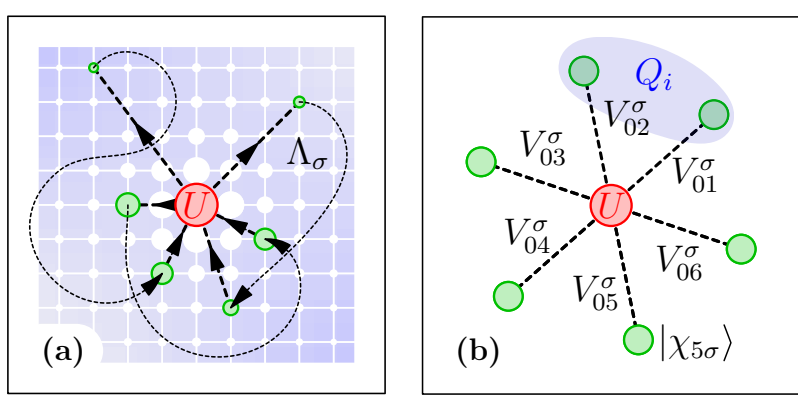

FIG. 1. (Color online) (a) DMFT impurity problem according to the action of Eq. (3). Arrows illustrate the effect of the twotime hybridization function $\Lambda_{\sigma}\left(t, t^{\prime}\right)$, which describes all possible processes where a particle of spin $\sigma$ jumps at time $t^{\prime}$ from the interacting impurity site (red) to a lattice site $i$ (green), propagates forward or backward in time from site $i$ to site $j$, and at a time $t$ jumps back to the impurity. (b) Representation of the DMFT bath by a single-impurity Anderson model (SIAM) with six bath orbitals and hopping matrix elements $V_{0 l}^{\sigma}, l \geqslant 1$. The variable $Q_{1}$ illustrates the combination of physical degrees of freedom into combined modes according to the MCTDH ansatz of Eq. (19).

where energies (times) are measured in terms of the hopping (inverse hopping) amplitude $(\hbar=1)$. For practical material simulations, $t_{i j}$ and $U$ could in principle be determined in an $a b$ initio manner, which is standard for DMFT simulations at equilibrium [29-31]. The unit of time $\hbar /\left|t_{i j}\right|$ would be between 20 fs for narrow-band organic Mott insulators [2] and a few femtoseconds for transition metal oxides with a bandwidth in the electron volt range [32].

\section{A. Nonequilibrium DMFT and Hamiltonian-based impurity solvers}

The central task of nonequilibrium DMFT based on the Keldysh formalism [33] is to compute the local contourordered Green's function,

$$
G_{\sigma}\left(t, t^{\prime}\right)=-i\left\langle T_{\mathcal{C}} c_{\sigma}(t) c_{\sigma}^{\dagger}\left(t^{\prime}\right)\right\rangle_{S_{\text {loc }}},
$$

of an effective single-site impurity model, which exactly replaces the original translationally invariant lattice problem, (1), in the limit of an infinite lattice coordination (and represents an approximation for finite dimensions). We follow Ref. [9] for the notation of contour-ordered functions, i.e., time arguments lie on the L-shaped Keldysh contour $\mathcal{C}$, and $\left\langle T_{\mathcal{C}} \ldots\right\rangle_{S_{\mathrm{loc}}} \equiv \operatorname{Tr}\left[T_{\mathcal{C}} e^{S_{\mathrm{loc}}} \ldots\right] / \operatorname{Tr}\left[T_{\mathcal{C}} e^{S_{\mathrm{loc}}}\right]$ denotes the contourordered expectation value. The action $S_{\mathrm{loc}}$ of the effective model is illustrated in Fig. 1(a) and is given by

$$
\begin{aligned}
S_{\mathrm{loc}}= & -i \int_{\mathcal{C}} d t\left[U(t)\left(n_{\uparrow}(t)-\frac{1}{2}\right)\left(n_{\downarrow}(t)-\frac{1}{2}\right)-\mu \sum_{\sigma} n_{\sigma}(t)\right] \\
& -i \int_{\mathcal{C}} \int_{\mathcal{C}} d t d t^{\prime} \sum_{\sigma} \Lambda_{\sigma}\left(t, t^{\prime}\right) c_{\sigma}^{\dagger}(t) c_{\sigma}\left(t^{\prime}\right),
\end{aligned}
$$

where the first part contains the Hamiltonian of an isolated site of the original lattice at a chemical potential $\mu$, and the second part connects the site to a noninteracting continuous bath which, at nonequilibrium, is defined by the hybridization function $\Lambda_{\sigma}\left(t, t^{\prime}\right)$. In single-site DMFT, the bath must be 
determined self-consistently from the equations of motion of $\Lambda_{\sigma}$, which depend on the impurity Green's function $G_{\sigma}\left(t, t^{\prime}\right)$ and the time-dependent hopping parameters $t_{i j}(t)$. In the simplest case, for a Bethe lattice in the limit of an infinite coordination number $Z$ with nearest-neighbor hopping (i.e., semielliptical density of states), the bath is characterized by a self-consistency relation of closed form [34],

$$
\Lambda_{\sigma}\left(t, t^{\prime}\right)=v(t) G_{\sigma}\left(t, t^{\prime}\right) v\left(t^{\prime}\right),
$$

where the hopping matrix elements in Eq. (1) are rescaled according to $t_{i j}(t) \rightarrow v(t) / \sqrt{Z}$.

Unfortunately, the DMFT action of the form (3) does not allow for a direct solution of the impurity problem with Hamiltonian-based methods. However, an optimal representation of $S_{\mathrm{loc}}$ in terms of a time-dependent impurity Hamiltonian with finitely many bath orbitals can be obtained by a suitable decomposition of the two-time hybridization function. Formally, such a mapping requires that all impurity correlation functions $\left\langle\mathcal{O}\left(t_{1}\right) \ldots\right\rangle$ are the same when computed with the action $S_{\text {loc }}$ or with the final impurity Hamiltonian $H^{\prime}(t)$, i.e.,

$$
\begin{aligned}
& \frac{\operatorname{Tr}\left(T_{\mathcal{C}}\left\{\exp \left(S_{\mathrm{loc}}\right) \mathcal{O}\left(t_{1}\right) \ldots\right\}\right)}{\operatorname{Tr}\left(T_{\mathcal{C}}\left\{\exp \left(S_{\mathrm{loc}}\right)\right\}\right)} \\
& \stackrel{\operatorname{Tr}\left(T_{\mathcal{C}}\left\{\exp \left(-i \int_{\mathcal{C}} d t H^{\prime}(t)\right) \mathcal{O}\left(t_{1}\right) \ldots\right\}\right)}{\operatorname{Tr}\left(T_{\mathcal{C}}\left\{\exp \left(-i \int_{\mathcal{C}} d t H^{\prime}(t)\right)\right\}\right)} .
\end{aligned}
$$

A particularly convenient mapping [14] becomes possible for the SIAM where $H^{\prime}=H_{\text {SIAM }}=H_{\text {imp }}+H_{\text {bath }}+H_{\text {hyb }}$ with

$$
\begin{aligned}
& H_{\mathrm{imp}}=-\mu \sum_{\sigma} n_{0 \sigma}+U(t)\left(n_{0 \uparrow}-\frac{1}{2}\right)\left(n_{0 \downarrow}-\frac{1}{2}\right), \\
& H_{\mathrm{bath}}=\sum_{l=1}^{L} \sum_{\sigma}\left(\epsilon_{l \sigma}-\mu\right) c_{l \sigma}^{\dagger} c_{l \sigma}, \\
& H_{\mathrm{hyb}}=\sum_{l=1}^{L} \sum_{\sigma}\left(V_{0 l}^{\sigma}(t) c_{0 \sigma}^{\dagger} c_{l \sigma}+\text { H.c. }\right) .
\end{aligned}
$$

Here, the impurity site is coupled in a star pattern by hopping processes of amplitude $V_{0 l}^{\sigma}(t)$ to $L$ individual noninteracting bath orbitals of energy $\epsilon_{l \sigma}$, and the operator $c_{l \sigma}\left(c_{l \sigma}^{\dagger}\right)$ annihilates (creates) an electron in a spin orbital $\left|\chi_{l \sigma}\right\rangle$ at bath site $l$ for $l>0$ and at the impurity site for $l=0$ [see illustration in Fig. 1(b)]. The hybridization function of the SIAM is given by

$$
\Lambda_{\sigma}^{\prime}\left(t, t^{\prime}\right)=\sum_{l=1}^{L} V_{0 l}^{\sigma}(t) g\left(\epsilon_{l \sigma}, t, t^{\prime}\right) V_{l 0}^{\sigma}\left(t^{\prime}\right),
$$

where $g\left(\epsilon, t, t^{\prime}\right)=-i\left[\theta_{\mathcal{C}}\left(t, t^{\prime}\right)-f(\epsilon)\right] e^{-i \epsilon\left(t-t^{\prime}\right)}$ is the Green's function of an isolated bath orbital, $f(\epsilon)=1 /\left(e^{\beta \epsilon}+1\right)$ denotes the Fermi distribution, and $\theta_{\mathcal{C}}$ is the contour step function. Since the exponential $e^{-i \epsilon\left(t-t^{\prime}\right)}$ can be absorbed into the time dependence of the parameters $V_{0 l}^{\sigma}(t)$, the problem of representing the DMFT action by the Hamiltonian, (6), has thus been reduced to a factorization of a "two-time matrix" $\Lambda_{\sigma}\left(t, t^{\prime}\right)$ in terms of time-dependent functions.

If the bath is initially decoupled from the impurity (this is commonly referred to as the atomic limit), the initial state of the system is entirely described in terms of the impurity density matrix, and $\Lambda_{\sigma}\left(t, t^{\prime}\right)$ is only nonzero for times $t, t^{\prime}>0$ on the real part of the contour. The parameters in the SIAM can then be obtained by demanding that the greater and lesser components of the original hybridization functions $\Lambda_{\sigma}\left(t, t^{\prime}\right)$ and $\Lambda_{\sigma}^{\prime}\left(t, t^{\prime}\right)$ of Eq. (7) are identical for all times $t$ and $t^{\prime}$. In practice, this leads to a matrix decomposition of $\Lambda_{\sigma}\left(t, t^{\prime}\right)$, where the matrix rank $N_{\mathrm{t}}$ is defined by the discretization of times $t$ and $t^{\prime}$ according to $0, \delta t, 2 \delta t, \ldots,\left(N_{\mathrm{t}}-1\right) \delta t$. By choosing the bath energies of the SIAM such that the occupations $f\left(\epsilon_{l \sigma}-\mu\right)$ are either 0 or 1 , the greater and lesser components can be decomposed independently of one another:

$$
\begin{aligned}
-i \Lambda_{\sigma}^{<}\left(t, t^{\prime}\right) & =\sum_{l=1}^{L / 2} V_{0 l}^{\sigma}(t)\left[V_{0 l}^{\sigma}\left(t^{\prime}\right)\right]^{*}, \\
i \Lambda_{\sigma}^{>}\left(t, t^{\prime}\right) & =\sum_{l=L / 2+1}^{L} V_{0 l}^{\sigma}(t)\left[V_{0 l}^{\sigma}\left(t^{\prime}\right)\right]^{*},
\end{aligned}
$$

where we have initially occupied the first half of the spin-orbitals $\left|\chi_{l \sigma}\right\rangle$ and left the other half empty (note that nonuniform partitions are also possible).

It is obvious that Eq. (8) holds, in general, only in the limit $L \rightarrow \infty$, ensuing from an infinite DMFT bath. However, appropriate representations can usually be obtained already for a rather small number of bath orbitals [16]. Furthermore, by using a low-rank Cholesky approximation in Eq. (8) one can guarantee that the representation of the hybridization function is always correct at short times such that a gradual increase in $L$ allows us to successively approach longer and longer simulation times [14]. Finally, we note that if impurity and bath hybridize already in the initial state [i.e., for $t \leqslant 0$, where the Hamiltonian $H(t)$ is time independent], the SIAM representation of the DMFT action, (3), requires additional bath sites which describe the time evolution of initial correlations; for details see also [14].

\section{B. Multiconfiguration time-dependent Hartree}

Practical applications of DMFT require an efficient solver for the time-dependent Schrödinger equation (TDSE) of the impurity model, (6). The MCTDH method [22,23,35,36], which has been applied to a variety of molecular quantum dynamics problems since its inception more than 20 years ago, is a general approach to efficiently solve the TDSE for multidimensional systems that tries to alleviate the exponential increase in computational effort with system size by a compact representation of the time-dependent state vector of the system. We first describe the original formulation of the MCTDH for systems of distinguishable degrees of freedom. The extension to indistinguishable particles (Sec. IIC) is then very similar and even uses essentially the same numerical implementation.

The standard wave function ansatz to solve the TDSE for a system with $f$ degrees of freedom reads

$$
\begin{aligned}
\left|\Psi\left(q_{1}, \ldots, q_{f}, t\right)\right\rangle & =\sum_{j_{1}=1}^{N_{1}} \ldots \sum_{j_{f}=1}^{N_{f}} C_{j_{1} \ldots j_{f}}(t) \prod_{\kappa=1}^{f}\left|\chi_{j_{\kappa}}^{(\kappa)}\left(q_{\kappa}\right)\right\rangle \\
& =\sum_{J} C_{J}(t)\left|\Xi_{J}\right\rangle,
\end{aligned}
$$


which expands the wave function as a sum of $D=\prod_{\kappa=1}^{f} N_{\kappa}$ Hartree products of one-dimensional primitive basis functions $\left|\chi_{j_{\kappa}}^{(\kappa)}\left(q_{\kappa}\right)\right\rangle$. For convenience, these functions are chosen orthonormal without loss of generality. Once a primitive basis has been selected, the time evolution of the system is fully determined by the set of time-dependent expansion coefficients $C_{j_{1} \ldots j_{f}}(t)$, which constitute a multidimensional tensor of rank $f$. Inserting Eq. (9) into the TDSE and multiplying from the left by $\left\langle\Xi_{L}\right|$ results in the linear matrix equation,

$$
i \dot{C}_{L}=\sum_{J}\left\langle\Xi_{L}|H| \Xi_{J}\right\rangle C_{J} .
$$

If, for simplicity, $N_{\kappa}=N$ for all $\kappa$, the standard approach has an exponential scaling $N^{f}$ in the number of expansion coefficients with the dimensionality and is therefore only practicable for a few degrees of freedom.

The MCTDH ansatz for the wave function reads

$$
\begin{aligned}
\left|\Psi\left(q_{1}, \ldots, q_{f}, t\right)\right\rangle & =\sum_{j_{1}=1}^{n_{1}} \ldots \sum_{j_{f}=1}^{n_{f}} A_{j_{1} \ldots j_{f}}(t) \prod_{\kappa=1}^{f}\left|\varphi_{j_{\kappa}}^{(\kappa)}\left(q_{\kappa}, t\right)\right\rangle \\
& =\sum_{J} A_{J}(t)\left|\Phi_{J}(t)\right\rangle,
\end{aligned}
$$

where $D_{A}=\prod_{\kappa=1}^{f} n_{\kappa}$ is the dimensionality of the $A$ vector $A_{i_{1} \ldots j_{f}}$ and the key difference from the standard ansatz is the introduction of time-dependent single-particle functions (SPFs) $\left|\varphi_{j_{\kappa}}^{(\kappa)}\left(q_{\kappa}, t\right)\right\rangle$, which are taken to be orthonormal for all times. The MCTDH equations of motion for the timedependent coefficients and SPFs are derived from the DiracFrenkel variation principle and read [22]

$$
\begin{aligned}
i \dot{A}_{J} & =\sum_{L}\left\langle\Phi_{J}|H| \Phi_{L}\right\rangle A_{L}, \\
i \dot{\varphi}^{(\kappa)} & =\left(1-P^{(\kappa)}\right)\left(\rho^{(\kappa)}\right)^{-1}\langle\mathbf{H}\rangle^{(\kappa)} \varphi^{(\kappa)} .
\end{aligned}
$$

Here a vector notation $\varphi^{(\kappa)}=\left(\left|\varphi_{1}^{(\kappa)}\right\rangle, \ldots,\left|\varphi_{n_{\kappa}}^{(\kappa)}\right\rangle\right)^{T}$ is used,

$$
P^{(\kappa)}=\sum_{j=1}^{n_{\kappa}}\left|\varphi_{j}^{(\kappa)}\right\rangle\left\langle\varphi_{j}^{(\kappa)}\right|
$$

is the projector on the space spanned by the SPFs for the $\kappa$ th degree of freedom, and $\langle\mathbf{H}\rangle^{(\kappa)}$ and $\rho^{(\kappa)}$ are mean fields and the density matrix. By defining single-hole functions $\left|\Psi_{l}^{(\kappa)}\right\rangle$ as linear combinations of Hartree products of $(f-1)$ SPFs without the SPFs for the $\kappa$ th degree of freedom $q_{\kappa}$,

$$
\begin{aligned}
\left|\Psi_{l}^{(\kappa)}\right\rangle= & \sum_{j_{1}} \ldots \sum_{j_{\kappa-1}} \sum_{j_{\kappa+1}} \ldots \sum_{j_{f}} A_{j_{1} \ldots j_{\kappa-1}} l j_{\kappa+1} \ldots j_{f} \\
& \times\left|\varphi_{j_{1}}^{(1)}\right\rangle \ldots\left|\varphi_{j_{\kappa-1}}^{(\kappa-1)}\right\rangle\left|\varphi_{j_{\kappa+1}}^{(\kappa+1)}\right\rangle \ldots\left|\varphi_{j_{f}}^{(f)}\right\rangle
\end{aligned}
$$

one can write $\langle\mathbf{H}\rangle^{(\kappa)}$ and $\rho^{(\kappa)}$ in compact forms as

$$
\langle H\rangle_{j l}^{(\kappa)}=\left\langle\Psi_{j}^{(\kappa)}|H| \Psi_{l}^{(\kappa)}\right\rangle
$$

and

$$
\begin{aligned}
\rho_{j l}^{(\kappa)}= & \left\langle\Psi_{j}^{(\kappa)} \mid \Psi_{l}^{(\kappa)}\right\rangle=\sum_{j_{1}} \ldots \sum_{j_{\kappa-1}} \sum_{j_{\kappa+1}} \ldots \sum_{j_{f}} \\
& \times A_{j_{1} \ldots j_{\kappa-1} j j_{\kappa+1} \ldots j_{f}}^{*} A_{j_{1} \ldots j_{\kappa-1} l j_{\kappa+1} \ldots j_{f}} .
\end{aligned}
$$

For a complete set of SPFs, $n_{\kappa}=N_{\kappa}$, one has $P^{(\kappa)}=1$ and hence $i \dot{\varphi}^{(\kappa)}=0$, such that Eqs. (12) correspond to the standard method of Eq. (10). With variationally optimal SPFs, the number of basis functions per degree of freedom can be kept smaller than the number of time-independent primitive functions. The number of coefficients in the $A$ vector still grows exponentially as $n^{f}$, but now to a smaller base (assuming equal $N_{\kappa}$ and $n_{\kappa}$ for all degrees of freedom, the number of time-dependent coefficients representing the MCTDH wave function is $\left.n^{f}+f N n\right)$. In the limit of all $n_{\kappa}=1$, the evolution is described by a single Hartree product of time-evolving SPFs, which corresponds to the time-dependent Hartree method. The advantage of the MCTDH method over the standard method lies in the fact that a much smaller number of differential equations has to be solved and the accuracy and cost of the calculation can be controlled by choosing the number of SPFs $n_{\kappa}$ for each degree of freedom. However, both Eqs. (12) are nonlinear, and $\left\langle\Phi_{J}|H| \Phi_{L}\right\rangle$ as well as the mean fields $\langle\mathbf{H}\rangle^{(\kappa)}$ must be rebuilt at each time step, which is usually the largest computational burden of MCTDH calculations.

Although other choices may be possible, the SPFs are often expanded on a set of time-independent orthogonal basis functions as in the standard approach, i.e.,

$$
\left|\varphi_{j_{\kappa}}^{(\kappa)}\left(q_{\kappa}, t\right)\right\rangle=\sum_{l_{\kappa}}^{N_{\kappa}} B_{j_{\kappa} l_{\kappa}}^{(\kappa)}\left|\chi_{l_{\kappa}}^{(\kappa)}\left(q_{\kappa}\right)\right\rangle .
$$

The MCTDH ansatz can then be regarded as a way to compactify the $C_{j_{1} \ldots j_{f}}$ tensor introduced in Eq. (9) as

$$
C_{j_{1} \ldots j_{f}}=\sum_{l_{1}}^{n_{1}} \ldots \sum_{l_{f}}^{n_{f}} A_{l_{1} \ldots l_{f}} \prod_{\kappa=1}^{f} B_{l_{\kappa} j_{\kappa}}^{(\kappa)} .
$$

This decomposition, which is graphically represented in Fig. 2(b), is known as the Tucker tensor decomposition [37] and has the same form as a matrix singular-value

(a)

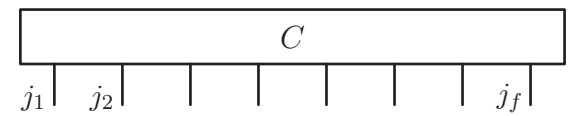

(b)

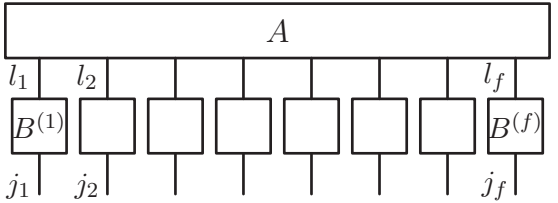

(c)

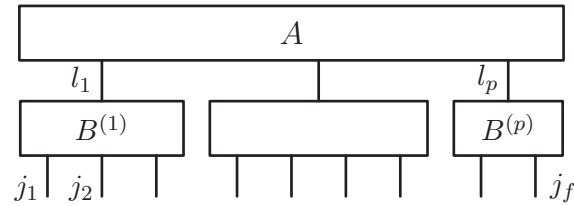

FIG. 2. From top to bottom, tensor networks representing the coefficients of the MCTDH ansätze of Eqs. (9), (18), and (20), respectively. Each box represents a tensor with as many indices as outgoing lines. A sum is performed over all indices at a line connecting two tensors. 
decomposition generalized to the multidimensional case [38]. The dimensionality of the $A$ vector can be further reduced by combining physical coordinates $q$ in fewer combined modes or logical coordinates $Q$. In this case the ansatz reads

$$
\begin{aligned}
\left|\Psi\left(q_{1}, \ldots, q_{f}, t\right)\right\rangle & \equiv\left|\Psi\left(Q_{1}, \ldots, Q_{p}, t\right)\right\rangle \\
& =\sum_{j_{1}}^{n_{1}} \ldots \sum_{j_{p}}^{n_{p}} A_{j_{1} \ldots j_{p}}(t) \prod_{\kappa=1}^{p}\left|\varphi_{j_{\kappa}}^{(\kappa)}\left(Q_{\kappa}, t\right)\right\rangle \\
& =\sum_{J} A_{J}(t)\left|\Phi_{J}(t)\right\rangle
\end{aligned}
$$

where $p$ is the number of MCTDH combined modes and the SPFs are now multidimensional functions. In terms of a more general tensorial decomposition similar to Eq. (18), the mode combination ansatz becomes [cf. Fig. 2(c)]

$$
C_{j_{1} \ldots j_{f}}=\sum_{l_{1}}^{n_{1}} \ldots \sum_{l_{p}}^{n_{p}} A_{l_{1} \ldots l_{p}} \prod_{\kappa=1}^{p} B_{l_{\kappa} ; J_{\kappa}}^{(\kappa)},
$$

where $J_{\kappa}$ refers to a multi-index collecting the $d_{\kappa}$ degrees of freedom, which are combined into one combined mode $\kappa$, i.e., $J_{1}=\left(j_{1}, \ldots, j_{d_{1}}\right), J_{2}=\left(j_{d_{1}+1}, \ldots, j_{d_{1}+d_{2}}\right), \ldots, J_{p}=$ $\left(j_{d_{1}+\ldots+d_{p-1}+1}, \ldots, j_{f}\right)$. The use of mode combination allows one to distribute the cost of the calculation in an optimal way between the propagation of the $A$ and $B$ coefficients (i.e., between the $A$ vector and the SPFs). Large combined modes lead to a small $A$ vector that can be efficiently propagated but result in the costly propagation of multidimensional SPFs. Providing a scheme for efficiently propagating multidimensional wave functions is what the MCTDH method does in the first place by introducing a multiconfigurational ansatz. A natural extension consists in expanding the multidimensional SPFs as sums of products of time-dependent basis functions of lower dimensionality, which is known as the ML-MCTDH [24-26]. In terms of the tensorial argument, it consists in decomposing the $B_{l_{\kappa} ; J_{\kappa}}^{(\kappa)}$ tensors in the same form as Eq. (18) or Eq. (20).

Computationally, MCTDH is most efficient when the system Hamiltonian is given by a sum of products of lowdimensional operators, as this immediately factorizes the matrix elements in Eqs. (12) into products of lower-dimensional integrals (note that the Hamiltonian and mean-field matrix elements need to be re-evaluated at every time step because they depend on the wave function via the time-dependent SPFs). For model Hamiltonians fulfilling this product form the (ML-)MCTDH method has been applied to thousands of degrees of freedom (e.g., [39]).

\section{MCTDH in second quantization representation}

Using Hartree products as the elementary configurations, the MCTDH framework introduced above describes the time evolution of distinguishable particles rather than the dynamics of fermionic or bosonic many-body states. Approaches which explicitly account for the exchange symmetry of the wave function are the MCTDH for fermions (MCTDHF) [40-44], which is based on a multiconfiguration expansion of the wave function in terms of Slater determinants built from time-dependent spin orbitals, and the bosonic version of the
MCTDH, in which the many-body configurations are taken to be permanents [45-47].

In order to solve the dynamics of indistinguishable spin-1/2 fermions in the SIAM one may use an MCTDHF expansion of the wave function or one can work in a second quantization representation (SQR), in which case the normal MCTDH machinery as introduced above for distinguishable degrees of freedom is applicable. We briefly introduce the MCTDHF alternative before discussing in more depth the SQR treatment used in this work. The MCTDHF scheme is readily obtained as a special case of Eq. (11) by using the same set of SPFs,

$$
\left|\varphi_{j \sigma}(t)\right\rangle=\sum_{l=1}^{M} B_{j \sigma, l}(t)\left|\chi_{l \sigma}\right\rangle,
$$

for all electronic coordinates and requiring that $A_{j_{1} \ldots j_{f}}$ is antisymmetric with respect to exchange of any two indices at all times [40]. For efficiency reasons, in actual implementations one primarily works with Slater determinants and uses the Slater-Condon rules to evaluate the one- and two-body matrix elements of the Hamiltonian. For the Hubbard model in the limit $U \rightarrow 0$, where all two-body matrix elements vanish, the dynamics is then trivially captured by a single Slater determinant with the SPFs evolving under the second line of Eq. (12) and the mean field being just the one-body Hamiltonian. Such a single-configuration description corresponds to the time-dependent Hartree-Fock scheme, which may still provide reasonable results for small $U$. In general, however, the problem becomes multiconfigurational in nature for interactions $U \neq 0$ and can become very hard for large $U$, requiring conceivably a huge set of Slater determinants to obtain convergent results.

Apart from using determinants (or permanents) in the multiconfiguration expansion it is, however, also possible to describe the dynamics of many-body systems of fermionic (bosonic) symmetry by working explicitly within the occupation number representation, such that the state vectors are members of Fock space instead of a Hilbert space. This scheme was introduced by Thoss and Wang under the name MCTDH in SQR (MCTDH-SQR) [24]. As we use the MCTDH-SQR instead of the MCTDHF to solve the DMFT impurity problem, we briefly review the key points of the scheme and refer the interested reader to the original reference.

For $M$ spin orbitals, the basis of the Fock space is given by the collection of states

$$
\left|n_{1}, n_{2}, \ldots, n_{M}\right\rangle=\prod_{i=1}^{M}\left(c_{i}^{\dagger}\right)^{n_{i}}\left|0_{1}, 0_{2}, \ldots, 0_{M}\right\rangle
$$

where for fermions $n_{i}=0,1$ are the allowed occupations of the spin orbital $\left|\chi_{i}\right\rangle,\left|0_{1}, 0_{2}, \ldots, 0_{M}\right\rangle$ denotes the empty vacuum state, and $c_{i}^{\dagger}$ and $c_{i}$ are the fermionic creation and annihilation operators which satisfy the anticommutation relations,

$$
\begin{aligned}
& \left\{c_{i}, c_{j}^{\dagger}\right\} \equiv c_{i} c_{j}^{\dagger}+c_{j}^{\dagger} c_{i}=\delta_{i j}, \\
& \left\{c_{i}, c_{j}\right\}=\left\{c_{i}^{\dagger}, c_{j}^{\dagger}\right\}=0 .
\end{aligned}
$$

To apply the standard MCTDH formalism for distinguishable particles for the basis of Eq. (22) we represent all basis vectors $\left|n_{1}, n_{2}, \ldots, n_{M}\right\rangle$ as Hartree products of kets $\left|n_{i}\right\rangle$ for 
the occupation of each spin orbital,

$$
\left|n_{1}, n_{2}, \ldots, n_{M}\right\rangle=\left|n_{1}\right\rangle \otimes\left|n_{2}\right\rangle \otimes \ldots \otimes\left|n_{M}\right\rangle .
$$

Formally, this is done by performing an inverse Jordan-Wigner transformation of the fermionic degrees of freedom, i.e.,

$$
\begin{aligned}
& c_{j}^{\dagger}=\exp \left(+i \pi \sum_{k=1}^{j-1} c_{k}^{\dagger} c_{k}\right) S_{j}^{+}, \\
& c_{j}=\exp \left(-i \pi \sum_{k=1}^{j-1} c_{k}^{\dagger} c_{k}\right) S_{j}^{-},
\end{aligned}
$$

and

$$
n_{j}=c_{j}^{\dagger} c_{j}=S_{j}^{z}+\frac{1}{2},
$$

where $S_{j}^{+}=\frac{1}{2}\left(\sigma_{j}^{x}+i \sigma_{j}^{y}\right), S_{j}^{-}=\frac{1}{2}\left(\sigma_{j}^{x}-i \sigma_{j}^{y}\right)$, and $S_{j}^{z}=\frac{1}{2} \sigma_{j}^{z}$ are the standard spin (ladder) operators with Pauli matrices $\sigma_{j}^{x}, \sigma_{j}^{y}$, and $\sigma_{j}^{z}$. In this way all operators acting in Fock space are mapped to products of $2 \times 2$ matrices and the occupation number states of a spin orbital $\left|\chi_{j}\right\rangle$ become states of a twodimensional vector space:

$$
\begin{array}{r}
\left|n_{j}=0\right\rangle \Leftrightarrow\left(\begin{array}{l}
0 \\
1
\end{array}\right), \\
\left|n_{j}=1\right\rangle \Leftrightarrow\left(\begin{array}{l}
1 \\
0
\end{array}\right) .
\end{array}
$$

Furthermore, regarding Eq. (9), the expansion coefficients $C_{n_{1} \ldots n_{M}}$ then do not fulfill any particular antisymmetry relation upon exchange of their indices and thus can be compactified according to Eq. (20).

It is clear that any operator in second quantization, such as the Hamiltonians in Eqs. (1) and (6), consists of a sum of products of terms acting on one degree of freedom only, where the degrees of freedom are the occupation numbers of each spin-orbital. All the exchange symmetry logic is contained in the products of sign-change operators $S_{Q}$ acting on the degrees of freedom in front of position $P$, where a particle is being either created or annihilated, i.e., the (anti-)symmetry properties of the system are carried by the operator and not by the state vector as is the case in first quantization.

In the second quantization treatment, the one-electron terms of Hamiltonian (1), $t_{i j} c_{i \sigma}^{\dagger} c_{j \sigma}$, change two occupation numbers (and therefore two degrees of freedom) at once in the process of describing electron hopping, which is the definition of a two-body term. In contrast, electron repulsion terms of the form $U n_{i \uparrow} n_{i \downarrow}$ do not change any occupation numbers. The application of such a term to any ket in occupation number representation returns either the same ket or 0 . Therefore these terms are not responsible for any dynamics in this representation and simply shift the total energy of some occupation number state kets with respect to others. The different role of the electron hopping and electron repulsion terms in the first quantization and Fockspace descriptions is irrelevant when exact (and therefore equivalent) calculations are performed using either or the other representation. This difference, however, becomes of utmost importance when multiconfigurational (or, in general, tensor contraction) schemes are attempted. Cases with $t \gg U$ will benefit from a first quantization (MCTDHF) approach where the degrees of freedom are the positions of each electron in the lattice. Cases with $t \ll U$ will be efficient in a Fock-space description (i.e., in MCTDH-SQR) where the degrees of freedom are the occupation numbers in the occupation number representation. One can thus think of a general framework based on the MCTDH method, which could efficiently treat the SIAM (and other lattice models) in the whole parameter regime from weak to strong on-site Coulomb interactions. As the present study focuses mainly on strong coupling, it emphasizes that the MCTDH-SQR can certainly be applied more advantageously than the MCTDHF in this regime.

On the other hand, it remains for future work to investigate the applicability of an MCTDHF solver to the SIAM of DMFT. In practice, virtually any MCTDH implementation for distinguishable particles with the possibility of mode combination or ML-MCTDH, e.g., the Heidelberg MCTDH package used here [35], can perform MCTDH-SQR calculations without further modification. All that needs to be done is to define the corresponding system Hamiltonian making use of the representation of second quantization operators as (products of) $2 \times 2$ matrices according to the rules introduced above.

\section{RESULTS AND DISCUSSION}

In this section, we apply the MCTDH-SQR method as impurity solver and evaluate its performance on the basis of the computational cost. In Sec. III A, we outline the procedure for a simple test bath and compute the time-dependent wave function of the corresponding SIAM including $L$ bath sites and $N=N_{\uparrow}+N_{\downarrow}=L+1$ fermions for various on-site interactions $U$. In Sec. III C, we discuss the self-consistency and illustrate the computation of the two-time impurity Green's function.

\section{A. Model setup}

To assess the performance of the MCTDH-SQR for a time-dependent impurity problem which is representative for a DMFT calculation, we solve an impurity model which is suddenly coupled to a bath with semielliptical density of states; i.e., we choose a hybridization function $\Lambda_{\sigma}\left(t, t^{\prime}\right)=$ $v(t) g_{\sigma}\left(t, t^{\prime}\right) v\left(t^{\prime}\right)$, where the coupling $v(t)$ to the bath is given by a Heavyside step function, and $g_{\sigma}$ is the equilibrium Green's function of the uncoupled bath,

$$
g_{\sigma}^{\gtrless}\left(t, t^{\prime}\right)=\mp i \int d \omega f \gtrless(\omega) A(\omega) e^{-i \omega\left(t-t^{\prime}\right)},
$$

with $f^{<}(\omega)=f(\omega)=1 /\left(e^{\beta \omega}+1\right), f^{>}(\omega)=1-f(\omega)$, and $A(\omega)=\frac{1}{2 \pi} \sqrt{4-\omega^{2}}$. Furthermore, we set the temperature to $T=\beta^{-1}=1$, which corresponds to the generic situation where the time scale on which the bath loses its memory is of the order of a few hopping times. The complex hopping parameters $V_{0 l}^{\sigma}(t)$ in the SIAM then follow from a low-rank Cholesky decomposition of $\Lambda_{\sigma}\left(t, t^{\prime}\right)$ [14]. As an illustration, Fig. 3 shows the resulting hopping parameters for a setup with $L=4$ bath sites on a time window up to $t=10$; the time discretization comprises $n_{t}=500$ time steps.

For the MCTDH-SQR setup, we group two bath sites (i.e., four bath spin orbitals corresponding to four degrees of freedom) into one combined mode. As each spin orbital can be 


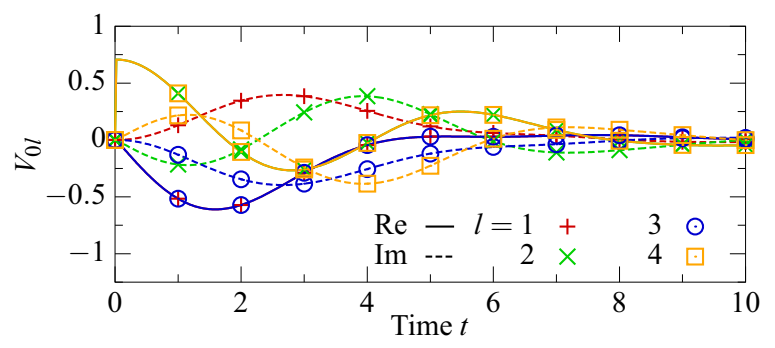

FIG. 3. (Color online) Time evolution of the complex hopping matrix elements $V_{0 l}(t)=V_{0 l}^{\uparrow}(t)=V_{0 l}^{\downarrow}(t)$ for a SIAM with $L=4$ bath orbitals and a reference bath which is governed by the equilibrium Green's function of Eq. (28) with inverse temperature $\beta=1$.

in either state $|0\rangle$ (empty) or state $|1\rangle$ (occupied), the span with $2^{4}=16$ SPFs represents the full Fock space. The impurity is left as a separate mode, which will, in practice, always be described with the maximum of $2^{2}=4$ SPFs. Starting from the atomic limit, the impurity site is initially decoupled from the bath and is occupied by a single up- or down-spin electron. Consistent with the decomposition scheme outlined in Sec. II A, the bath orbitals have different initial populations: The first half is doubly occupied, whereas the second half is empty ( $L$ even).

Assuming a SIAM with $N_{\uparrow}=L / 2+1$ spin-up particles and $N_{\downarrow}=L / 2$ spin-down particles, the dimension of the Hilbert space of the SIAM is given by

$$
D_{\mathrm{H}}=\left(\begin{array}{c}
L+1 \\
L / 2+1
\end{array}\right)\left(\begin{array}{c}
L+1 \\
L / 2
\end{array}\right) .
$$

On the other hand, the $A$ vector $A_{j_{1} \ldots j_{p}}$ in the MCTDH ansatz of Eq. (19) has dimension

$$
D_{\mathrm{A}}=2^{2}\left(N_{\mathrm{SPF}}^{\mathrm{b}}\right)^{2 L / N_{\mathrm{CM}}^{\mathrm{b}}}
$$

for an orbital partition scheme with $N_{\mathrm{CM}}^{\mathrm{b}}$ bath spin orbitals in a combined mode and each combined mode being represented by $N_{\text {SPF }}^{\mathrm{b}}$ SPFs (as stated above, the impurity degrees of freedom are treated in a single separate mode and are accounted for by the factor $2^{2}$ ). In Fig. 4 we observe that (despite the exponential scaling of the configuration space with $L$ ) application of the

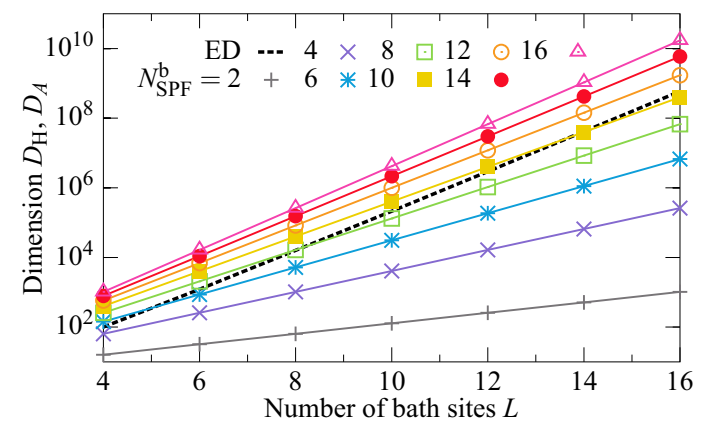

FIG. 4. (Color online) Hilbert-space dimension of the SIAM as a function of the number of bath sites $L$ (dashed black line) and dimensionality of the corresponding $A$ vectors in MCTDH-SQR (colored lines) for a setup where four bath spin orbitals are treated in a combined mode $\left(N_{\mathrm{CM}}^{\mathrm{b}}=4\right)$ and $N_{\mathrm{SPF}}^{\mathrm{b}}$ single-particle functions are involved [cf. Eq. (30)].
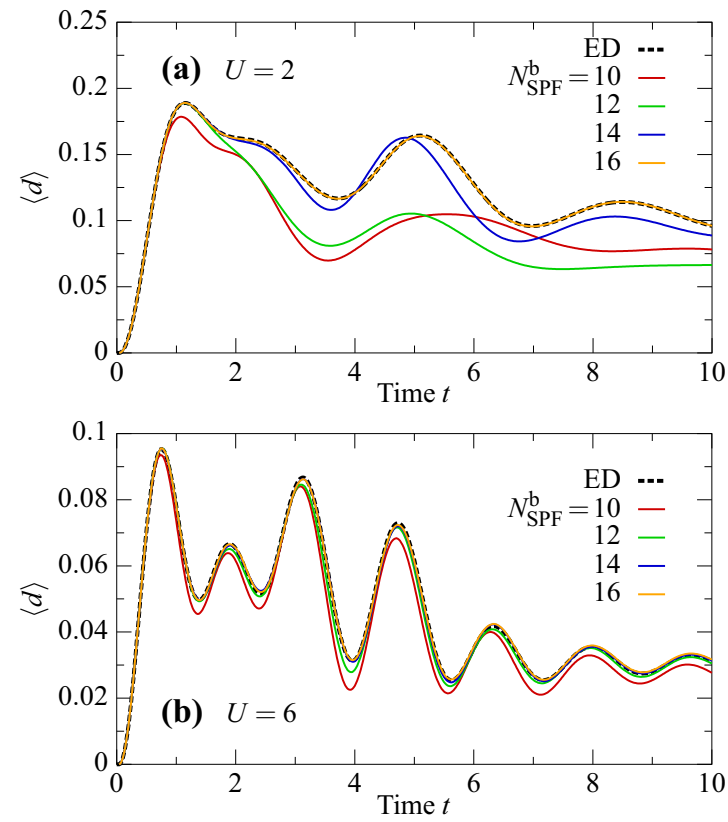

FIG. 5. (Color online) Time-dependent double occupancy $\langle d\rangle(t)$ of the impurity site for a SIAM with $L=4$ bath orbitals at (a) $U=2$ and (b) $U=6$, calculated by exact diagonalization (ED) and MCTDH-SQR with various numbers of SPFs $\left(N_{\mathrm{SPF}}^{\mathrm{b}}\right)$.

MCTDH-SQR can become favorable compared to ED for specific numbers of SPFs $N_{\mathrm{SPF}}^{\mathrm{b}}$ at fixed $L$, provided that the relevant observables of the impurity model are satisfactorily resolved in time. Note also that for large numbers of SPFs the size of the $A$ vector can exceed the size of the Hilbert space of the SIAM. This is due to the fact that the MCTDH-SQR is defined in the whole Fock space and an exact calculation corresponds to the sum of all Hilbert space sizes corresponding to all possible occupations.

\section{B. Comparison to exact diagonalization}

\section{Time evolution of the double occupancy}

To examine the quality of the MCTDH ansatz of the SIAM for different numbers of SPFs, we compute the time-dependent impurity double occupancy

$$
\langle d\rangle(t)=\left\langle\Psi(t)\left|n_{0 \uparrow} n_{0 \downarrow}\right| \Psi(t)\right\rangle
$$

for various sizes $L$ of the bath and different on-site interactions and compare it to exact reference data which are obtained by ED. In Figs. 5(a) and 5(b), we show MCTDH data for the SIAM with four bath sites at $U=2$ and $U=6$. In both cases, the MCTDH results for $N_{\mathrm{SPF}}^{\mathrm{b}}=16$ (orange lines) correspond to the time-dependent full configuration interaction result and thus lie perfectly on top of the ED curves. Since the dynamics starts from the atomic limit with a singly occupied impurity at $t=0$, the double occupation is initially 0 and then becomes finite and oscillatory; note that the density on the impurity site is a constant of motion by construction of the complex hopping matrix elements $V_{0 l}^{\sigma}(t)$. For $N_{\mathrm{SPF}}^{\mathrm{b}}<16$ the MCTDH results are approximate, and we generally find that convergence towards ED (by increasing the number of SPFs) is harder to reach as $U$ decreases. This behavior can be 
attributed to the fact that, during the time evolution at small $U$, the intersite hopping of electrons [i.e., the influence of $H_{\text {hyb }}$ in Eq. (6)] is more pronounced. Consequently, the wave function expands to a larger area in configuration space, which requires an increased number of time-adjusted SPFs $\left|\varphi_{j}(t)\right\rangle$ to optimally cover the support of $|\Psi(t)\rangle$. For strong coupling (large $U$ ), on the contrary, the wave function implies relatively weak intercoordinate correlation such that convergence can be reached more rapidly.

In summary, we expect that the MCTDH method can accurately capture the time evolution of the nonequilibrium impurity model in the moderate- to strong-coupling regime, where $U$ is larger than the kinetic energy. Moreover, it is important to note that the partition of spin orbitals into combined physical modes can affect the performance of the MCTDH [26]. When a combined mode contains both initially empty and initially unoccupied bath orbitals, the initial phase of the dynamics already involves a larger number of electronic configurations. Thus one may need a higher-dimensional basis to achieve observables of similar quality. A more favorable partition scheme is to group spin orbitals with the criterion that all bath spin orbitals of a combined mode are initially either empty or fully occupied. This guarantees that only a small set of possible electronic configurations can be accessed within the projected Fock space of a certain combined mode.

\section{Increase in configuration space with time}

We now attempt to estimate the size of the configuration space needed to access a certain maximum time. For this analysis we restrict ourselves to the case of strong coupling, where MCTDH converges most rapidly $(U=10)$. The configuration space is determined by two contributions: (i) the number of bath orbitals $L\left(t_{\max }\right)$ needed to accurately represent the dynamics up to $t=t_{\max }$ (cf. Sec. II A) and (ii) a possible reduction of the configuration space with respect to $D_{\mathrm{H}}\left(L\left(t_{\max }\right)\right)$ by MCTDH-SQR.

We first determine the configuration space needed within the ED approach. Throughout Fig. 6, the solid black line indicates the dynamics for the bath which is approximated by 14 sites, which is the largest system size accessible with ED in our implementation. Comparing these reference data with ED results for smaller $L$ (e.g., the dashed black lines in Figs. 6(a) and 6(b) for $L=10$ and $L=12$, respectively), we can extract a maximum physical time $t_{\max }(L)$ which can be reached in the calculation with a certain computational effort, measured by the corresponding Hilbert-space dimension $D_{\mathrm{H}}\left(L\left(t_{\max }\right)\right)$. The colored symbols in Fig. 7 indicate exponential scaling between $t_{\max }$ and $D_{\mathrm{H}}$ for ED, where $t_{\max }$ is determined by allowing for a maximum deviation of $1 \%$ [(red) crosses] and 10\% [(orange) squares] from the $L=14$ reference data.

From the plot it becomes clear that it is exponentially hard to reach long times with a Hamiltonian-based representation of a DMFT bath. Therefore it is an interesting question whether a MCTDH partition scheme with fewer and optimally timeevolving SPFs can lead to a more favorable scaling behavior. We obtain indications for this by analyzing the MCTDH results in Fig. 6 for a minimum number of SPFs for which $\langle d\rangle(t)$ is still satisfactorily described within an error of about $5 \%$. While in Figs. 6(a) and 6(b) we can directly compare to the
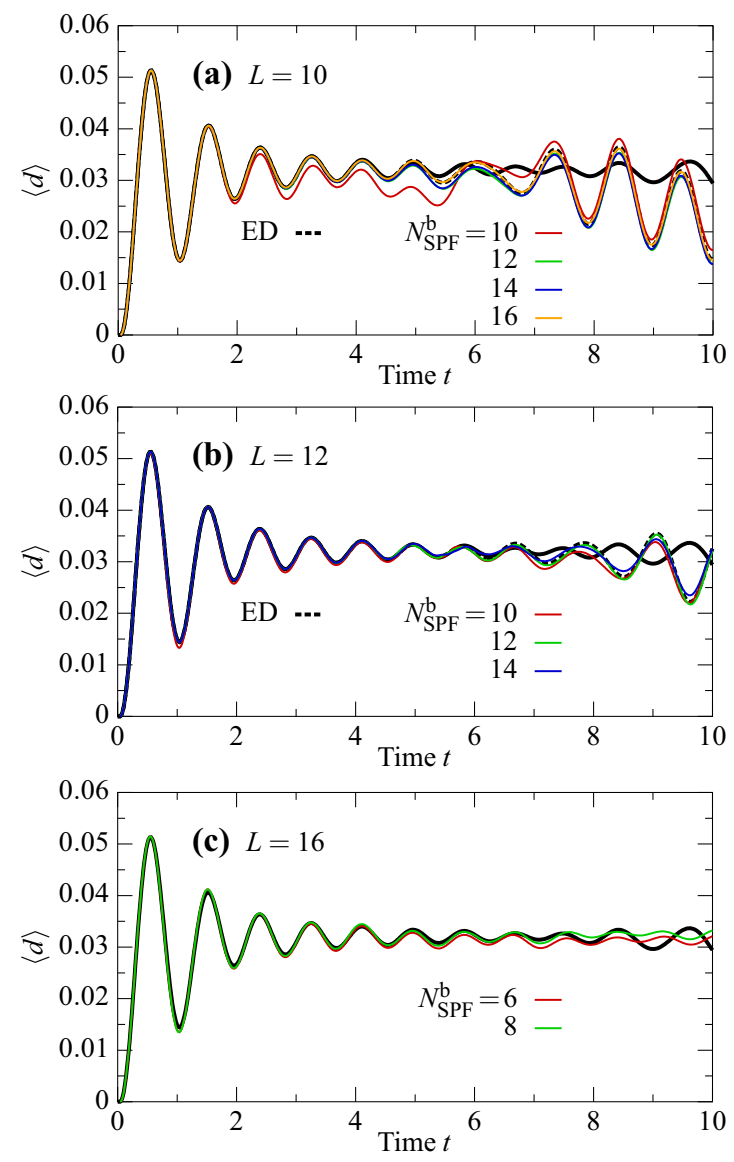

FIG. 6. (Color online) Comparison of MCTDH-SQR with four combined modes $\left(N_{\mathrm{CM}}=4\right)$ and $N_{\mathrm{SPF}}$ single-particle functions to exact diagonalization (ED) at an on-site interaction of $U=10$. Shown is the time-dependent impurity double occupancy $\langle d\rangle(t)$ for a SIAM with (a) $L=10$, (b) $L=12$, and (c) $L=16$ bath orbitals. The solid black line, showing the ED result for $L=14$, acts as a reference to determine the maximum time $t_{\max }$ in Fig. 7 .

corresponding ED result with the same number of bath orbitals (see dashed black lines), in Fig. 6(c) we only have the $L=14$ data as reference; here we estimate a maximum time of about $t_{\text {max }}=9$ up to which the oscillation of the double occupancy for $N_{\mathrm{SPF}}^{\mathrm{b}}=8$ is still decaying as a function of time.

The result of the analysis is presented by the black circles labeled $\left(L, N_{\mathrm{SPF}}^{\mathrm{b}}\right)$ in Fig. 7. Indeed, we find a deviating scaling for MCTDH which roughly follows the delineated thick dashed gray line as a function of $t_{\max }$. With eight SPFs, the calculation for $L=16$ also marks the first point where the size of the MCTDH $A$ vector $\left(D_{A} \approx 6.7 \times 10^{7}\right)$ is smaller than the size of the corresponding Hilbert space dealt with in the ED $\left(D_{\mathrm{H}} \approx 5.9 \times 10^{8}\right)$.

\section{Impurity Green's function}

For successful implementation as out-of-equilibrium impurity solver, the MCTDH method must be capable of accessing the two-time Green's function $G_{0 \sigma}\left(t, t^{\prime}\right)$ at the impurity site of the SIAM, from which the hybridization function is determined in a self-consistent manner. From this local Green's function one can then also obtain, e.g., the self-energy of 


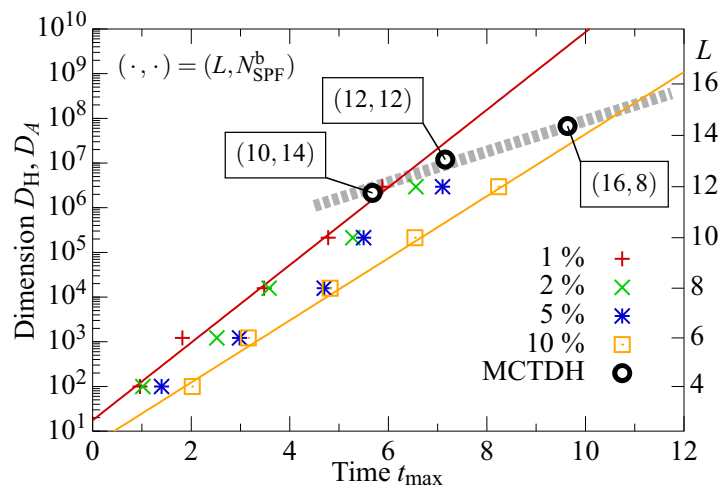

FIG. 7. (Color online) Numerical complexity of the time evolution to a given time $t_{\max }$ in the SIAM, measured in terms of the number $D$ of parameters in the wave-function ansatz [i.e., the required Hilbert-space dimension $D_{\mathrm{H}}(L)$ for exact diagonalization and the size of the tensor $D_{A}\left(L ; N_{\mathrm{SPF}}\right)$ in $\left.\mathrm{MCTDH}\right]$. To determine the maximum time $t_{\max }$ which can be accurately simulated for a given configuration ( $L, N_{\mathrm{SPF}}$ ), and hence with a given $D$, we require the error in the double occupation to be lower than a given bound $\epsilon$ [see Figs. (a)-6(c)]: yellow and red symbols and lines correspond to exact diagonalization and an $\epsilon$ value of $1 \%$ and $10 \%$, respectively. Black circles correspond to MCTDH and $\epsilon=5 \%$.

the system, the time-dependent momentum distribution, or spectroscopic observables of pump-probe experiments [9]. We emphasize that time- and frequency-resolved spectroscopic quantities such as the photoemission spectrum with an intrinsic Heisenberg-limited time-energy uncertainty are obtained from the Green's function $G\left(t, t^{\prime}\right)$ in the time window $t_{p}-\Delta t \leqslant$ $t, t^{\prime} \leqslant t_{p}+\Delta t$ of width $\Delta t$ around a probe time $t_{p}[48,49]$, so that these quantities can be obtained exactly from the solution of a SIAM with finitely many bath sites.

To demonstrate the general procedure and its feasibility within the MCTDH-SQR we follow Ref. [14] and consider the real-time dynamics of the Hubbard model on the Bethe lattice, starting from the atomic limit and from a zerotemperature initial state $(T=0)$. More precisely, we fix the on-site interaction to $U=4$ and study the dynamics of the paramagnetic phase at half-filling when the nearest-neighbor hopping in the infinite-dimensional lattice is ramped up from 0 to $v\left(t_{1}\right)=1$ with a cosine-shaped profile [see the dotted (red) line in Fig. 8(d)]; in the Hubbard Hamiltonian, (1), we thus consider $t_{i j}(t)=\delta_{\langle i j\rangle} v(t) / \sqrt{Z}$ in the limit of infinite coordination number $Z$.

The DMFT action of the lattice Hubbard model is mapped onto a SIAM with an initial state as described in Sec. III A; i.e., it contains an equal number of empty and doubly occupied bath sites with energy $\epsilon_{l}=0$ and a singly occupied impurity. The hopping parameters $V_{0 l}^{\sigma}(t)$ are spin independent and are determined self-consistently via the bath hybridization function $\Lambda_{\sigma}\left(t, t^{\prime}\right)=v(t) G_{0 \sigma}\left(t, t^{\prime}\right) v\left(t^{\prime}\right)$, where $G_{0 \uparrow}=G_{0 \downarrow}$ for all times on the contour. To generate an initial guess for $\Lambda_{\sigma}\left(t, t^{\prime}\right)$ we use the Green's function of Eq. (28); compare with Fig. 8(a).

Given the time-dependent MCTDH wave function $|\Psi(t)\rangle$ of the SIAM for $N=N_{\uparrow}+N_{\downarrow}$ particles, the two independent (lesser and greater) components of the impurity Green's
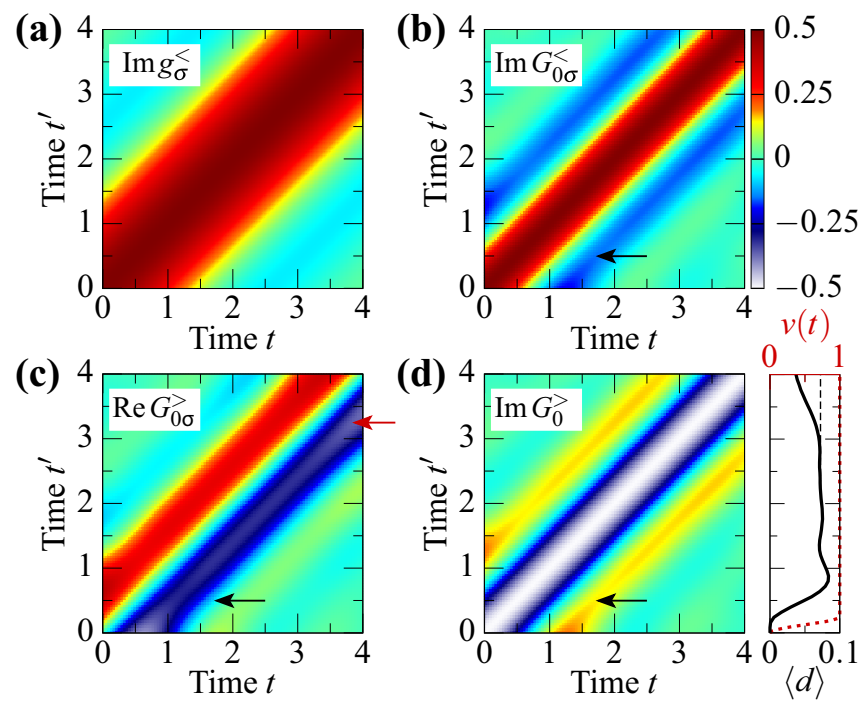

FIG. 8. (Color online) (a) Imaginary part of the Green's function $g_{\sigma}^{<}\left(t, t^{\prime}\right)$ of Eq. (28), which is used to compute the initial guess for the hybridization function in the first DMFT iteration. (b)-(d) Selfconsistent results for the local impurity Green's function $G_{0 \sigma}\left(t, t^{\prime}\right)$ as obtained from an MCTDH-SQR calculation with $L=8$ bath orbitals in the single-impurity Anderson model; the on-site Coulomb repulsion is $U=4$ (note that $G_{0 \sigma}^{>}=-G_{0 \sigma}^{<}$because of particle-hole symmetry). Black arrows indicate the early time domain, where the transient dynamics due to the switch-on of hopping is most pronounced. The gray (red) arrow in (c) points to the formation of small artifacts in the final "steady" state which are due to the representation of the DMFT bath with finitely many bath orbitals. Furthermore, in (d), the solid black line shows the time evolution of the double occupation $\langle d\rangle$ in the system, and the dotted (red) line indicates the switch-on of hopping.

function, $G_{0 \sigma}^{>}$and $G_{0 \sigma}^{<}$, can be computed as the overlaps

$$
\begin{aligned}
& G_{0 \sigma}^{>}\left(t, t^{\prime}\right)=-i\left\langle\Psi(t) \mid \Phi^{>}\left(t, t^{\prime}\right)\right\rangle, \\
& G_{0 \sigma}^{<}\left(t, t^{\prime}\right)=i\left\langle\Psi\left(t^{\prime}\right) \mid \Phi^{<}\left(t^{\prime}, t\right)\right\rangle,
\end{aligned}
$$

where the states $\left|\Phi^{\gtrless}\left(t, t^{\prime}\right)\right\rangle$ are defined by $\left|\Phi^{>}\left(t, t^{\prime}\right)\right\rangle=$ $c_{0 \sigma} U\left(t, t^{\prime}\right) c_{0 \sigma}^{\dagger}\left|\Psi\left(t^{\prime}\right)\right\rangle, \quad\left|\Phi^{<}\left(t, t^{\prime}\right)\right\rangle=c_{0 \sigma}^{\dagger} U\left(t, t^{\prime}\right) c_{0 \sigma}\left|\Psi\left(t^{\prime}\right)\right\rangle$ and $U\left(t, t^{\prime}\right)=T_{\mathrm{t}} e^{-i \int_{t^{\prime}}^{t} d s H^{\prime}(s)}$ denotes the time-evolution operator for the impurity model, (6). In practice, we evaluate the two-time Green's functions as

$$
\begin{aligned}
& G_{0 \sigma}^{>}\left(t, t^{\prime}\right)=-i\left\langle\Xi^{>}(t) \mid \Xi^{>}\left(t^{\prime}\right)\right\rangle, \\
& G_{0 \sigma}^{<}\left(t, t^{\prime}\right)=i\left\langle\Xi^{<}\left(t^{\prime}\right) \mid \Xi^{<}(t)\right\rangle,
\end{aligned}
$$

where $\left|\Xi^{>}(t)\right\rangle=U(0, t) c_{0 \sigma}^{\dagger}|\Psi(t)\rangle$ and $\left|\Xi^{<}(t)\right\rangle=U(0, t) c_{0 \sigma}$ $|\Psi(t)\rangle$ are the associated $(N+1)$ - and $(N-1)$-particle wave functions.

As the half-filled Hubbard model we start from is particlehole symmetric, but the SIAM with spin-imbalanced occupation is not, we use an adapted initial state which is a superposition of two degenerate states: One has a spin-up electron occupying the impurity site, and the other has a spin-down electron on the impurity site. An alternative scheme which we have also implemented to restore particle-hole symmetry is first to construct Green's functions $G^{A}\left(t, t^{\prime}\right)$ and 
$G^{B}\left(t, t^{\prime}\right)$ with interchanged particle numbers (i.e., $N_{\uparrow} \leftrightarrow N_{\downarrow}$ ) and then to average over the two Green's functions according to $G\left(t, t^{\prime}\right)=\frac{1}{2}\left[G^{A}\left(t, t^{\prime}\right)+G^{B}\left(t, t^{\prime}\right)\right]$.

In Figs. 8(b)-8(d) we present results for the self-consistent impurity Green's function where the hybridization function has been approximated in a time window $[0,4]$ by a SIAM with $L=8$ bath orbitals. We clearly see that while the density in the system, $\left\langle n_{\sigma}\right\rangle(t)=\operatorname{Im} G_{\sigma}^{<}(t, t)=0.5$, is a constant of motion, the time-off-diagonal components of the Green's function containing the spectral information develop as a function of the two times (see black arrows). Moreover, for times $t, t^{\prime} \gtrsim 1.5$ where the double occupation in the system approaches a stationary value, also the Green's functions attain a quasistatic structure as a function of the physical (center-of-mass) time $\left(t+t^{\prime}\right) / 2$.

To bring the results to convergence, we have implemented the self-consistency loop in two ways, either iterating on the full $\left(t, t^{\prime}\right)$ mesh or using the time propagation scheme described in Ref. [14]. While the former approach was simpler to implement, the latter is found to be much more efficient because the self-consistency is established for each time slice separately, allowing for essentially fewer iterations. Finally, we remark that the tiny changes in the Green's function at later times $(t \gtrsim 3)$ are due to the discretization of the DMFT bath with only eight bath sites; see, e.g., the gray (red) arrow in Fig. 8(c) and compare it to the time evolution of the double occupancy in Fig. 8(d), which also deviates from the steady state (dashed line) for times $t>3$.

\section{CONCLUSION}

In this work we have implemented and benchmarked a solution of the impurity problem of nonequilibrium DMFT based on the MCTDH method. The MCTDH method provides a variationally optimized representation of a time-dependent (fermionic or bosonic) wave function, which can reduce the dimension of the underlying basis function space by several orders of magnitude. Because the Hamiltonian representation of the DMFT action requires increasingly more bath orbitals for longer times, this compression of the wave function is a crucial feature to access the transient dynamics in exact nonequilibrium DMFT simulations beyond the current limit of very few inverse hopping times.

For the time-dependent SIAM, which represents the core component of the Hamiltonian-based DMFT approach out of equilibrium, we have been able to show that the MCTDH can indeed go beyond the capability of ED for sufficiently strong Coulomb interactions. For SIAMs with a small number of bath sites, an exact solution is more favorable than the MCTDH method, because the latter is implemented in SQR (MCTDH-SQR) where the state vector is defined in Fock space and contains redundant electronic configurations which are unphysical for simulating a system with a given number of electrons (and spin orbitals). For large systems, as needed to solve the DMFT problem at long times, the MCTDH method can become favorable. Using the concept of mode combination, we have provided the dynamics of a SIAM which describes a typical DMFT bath with $L=16$ bath orbitals and is not feasible to be solved by ED at a reasonable computational cost. This calculation marks the onset of a regime in which the state vector of MCTDH scales more favorably than the Hilbert space with the maximum physical time that can be accessed in the simulation.

Moreover, we have illustrated the feasibility of the MCTDH-SQR algorithm to yield the time-dependent observables as well as the self-consistent real-time Green's functions for a generic Hubbard-type lattice problem, which shows the potential of the method to act as full DMFT impurity solver. Although the efficiency to directly compute the Green's function with the standard Heidelberg MCTDH package for system sizes as large as $L \approx 16$ must still be proven, there are no conceptual difficulties to upscale the scope of the method.

In order to push the applicable regime of the MCTDHbased impurity solver to even larger SIAMs and hence to even longer time scales, it is very promising to extend the approach to the ML-MCTDH [24-26], by which the scaling barrier is expected to be more efficiently overcome. Thus, to find an optimal tree-tensor network decomposition of the fermionic SIAM wave function within the ML-MCTDH scheme is the main path for future work.

\section{ACKNOWLEDGMENTS}

The authors thank Michael Bonitz, Stephen Clark, Rainer Härtle, Marcus Kollar, Hans-Dieter Meyer, and Armin Scrinzi for intensive discussions. Z.L. and O.V. acknowledge financial support from the Hamburg Centre for Ultrafast Imaging (CUI).
[1] A. L. Cavalieri, N. Müller, T. Uphues, V. S. Yakovlev, A. Baltuška, B. Horvath, B. Schmidt, L. Blümel, R. Holzwarth, S. Hendel, M. Drescher, U. Kleineberg, P. M. Echenique, R. Kienberger, F. Krausz, and U. Heinzmann, Nature 449, 1029 (2007).

[2] S. Wall, D. Brida, S. R. Clark, H. P. Ehrke, D. Jaksch, A. Ardavan, S. Bonora, H. Uemura, Y. Takahashi, T. Hasegawa, H. Okamoto, G. Cerullo, and A. Cavalleri, Nature Phys. 7, 114 (2011).
[3] L. Perfetti, P. A. Loukakos, M. Lisowski, U. Bovensiepen, H. Berger, S. Biermann, P. S. Cornaglia, A. Georges, and M. Wolf, Phys. Rev. Lett. 97, 067402 (2006).

[4] S. Iwai, M. Ono, A. Maeda, H. Matsuzaki, H. Kishida, H. Okamoto, and Y. Tokura, Phys. Rev. Lett. 91, 057401 (2003).

[5] F. Schmitt, P. S. Kirchmann, U. Bovensiepen, R. G. Moore, L. Rettig, M. Krenz, J.-H. Chu, N. Ru, L. Perfetti, D. H. Lu, M. Wolf, I. R. Fisher, and Z.-X. Shen, Science 321, 1649 (2008). [6] U. Schollwöck, Rev. Mod. Phys. 77, 259 (2005). 
[7] P. Schmidt and H. Monien, arXiv:cond-mat/0202046.

[8] J. K. Freericks, V. M. Turkowski, and V. Zlatić, Phys. Rev. Lett. 97, 266408 (2006).

[9] H. Aoki, N. Tsuji, M. Eckstein, M. Kollar, T. Oka, and P. Werner, Rev. Mod. Phys. 86, 779 (2014).

[10] A. Georges, G. Kotliar, W. Krauth, and M. J. Rozenberg, Rev. Mod. Phys. 68, 13 (1996).

[11] M. Eckstein, M. Kollar, and P. Werner, Phys. Rev. Lett. 103, 056403 (2009).

[12] M. Eckstein and P. Werner, Phys. Rev. B 82, 115115 (2010).

[13] N. Tsuji and P. Werner, Phys. Rev. B 88, 165115 (2013).

[14] C. Gramsch, K. Balzer, M. Eckstein, and M. Kollar, Phys. Rev. B 88, 235106 (2013).

[15] A. Szabo and N. A. Ostlund, Modern quantum chemistry: Introduction to advanced electronic structure theory (Dover, Mineola, NY, 1996).

[16] K. Balzer and M. Eckstein, Phys. Rev. B 89, 035148 (2014).

[17] F. Hofmann, M. Eckstein, E. Arrigoni, and M. Potthoff, Phys. Rev. B 88, 165124 (2013).

[18] E. Arrigoni, M. Knap, and W. von der Linden, Phys. Rev. Lett. 110, 086403 (2013).

[19] F. Verstraete and J. I. Cirac, arXiv:cond-mat/0407066v1.

[20] G. Vidal, Phys. Rev. Lett. 99, 220405 (2007).

[21] V. Murg, F. Verstraete, and J. I. Cirac, Phys. Rev. A 75, 033605 (2007).

[22] M. H. Beck, A. Jäckle, G. A. Worth, and H.-D. Meyer, Phys. Rep. 324, 1 (2000).

[23] H.-D. Meyer, F. Gatti, and G. Worth (eds.), Multidimensional Quantum Dynamics: MCTDH Theory and Its Applications (Wiley-VCH, Weinheim, Germany, 2009).

[24] H. Wang and M. Thoss, J. Chem. Phys. 131, 024114 (2009).

[25] U. Manthe, J. Chem. Phys. 128, 164116 (2008).

[26] O. Vendrell and H.-D. Meyer, J. Chem. Phys. 134, 044135 (2011).

[27] K. F. Albrecht, H. Wang, L. Mühlbacher, M. Thoss, and A. Komnik, Phys. Rev. B 86, 081412 (2012).

[28] H. Wang and M. Thoss, J. Chem. Phys. 138, 134704 (2013).
[29] G. Kotliar and D. Vollhardt, Phys. Today 57, 53 (2004).

[30] A. I. Lichtenstein and M. I. Katsnelson, Phys. Rev. B 57, 6884 (1998).

[31] V. I. Anisimov, A. I. Poteryaev, M. A. Korotin, A. O. Anokhin, and G. Kotliar, J. Phys. Condens. Matter 9, 7359 (1997).

[32] M. Imada, A. Fujimori, and Y. Tokura, Rev. Mod. Phys. 70, 1039 (1998).

[33] L. V. Keldysh, Zh. Eksp. Teor. Fiz. 47, 1515 (1964) [Sov. Phys. JETP 20, 1018 (1965)].

[34] M. Eckstein, A. Hackl, S. Kehrein, M. Kollar, M. Moeckel, P. Werner, and F. A. Wolf, Eur. Phys. J. Special Topics 180, 217 (2010).

[35] G. A. Worth, M. H. Beck, A. Jäckle, and H.-D. Meyer, The MCTDH Package, Version 8.2 (Universität Heidelberg, Heidelberg, 2000); H.-D. Meyer, The MCTDH Package, Version 8.3 (2002), Version 8.4 (2007), Version 8.5 (2014). See http://mctdh.uni-hd.de.

[36] H.-D. Meyer, U. Manthe, and L. S. Cederbaum, Chem. Phys. Lett. 165, 73 (1990).

[37] L. Tucker, Psychometrika 31, 279 (1966).

[38] T. G. Kolda and B. W. Bader, SIAM Rev. 51, 455 (2009).

[39] H. Wang and M. Thoss, New J. Phys. 10, 115005 (2008).

[40] J. Caillat, J. Zanghellini, M. Kitzler, O. Koch, W. Kreuzer, and A. Scrinzi, Phys. Rev. A 71, 012712 (2005).

[41] M. Nest, Chem. Phys. Lett. 472, 171 (2009).

[42] I. S. Ulusoy and M. Nest, J. Chem. Phys. 136, 054112 (2012).

[43] J. M. Zhang and M. Kollar, Phys. Rev. A 89, 012504 (2014).

[44] D. Hochstuhl, C. M. Hinz, and M. Bonitz, Eur. Phys. J. Special Topics 223, 177 (2014).

[45] O. E. Alon, A. I. Streltsov, and L. S. Cederbaum, J. Chem. Phys. 127, 154103 (2007).

[46] O. E. Alon, A. I. Streltsov, and L. S. Cederbaum, Phys. Rev. A 77, 033613 (2008).

[47] L. Cao, S. Krönke, O. Vendrell, and P. Schmelcher, J. Chem. Phys. 139, 134103 (2013).

[48] J. K. Freericks, H. R. Krishnamurthy, and Th. Pruschke, Phys. Rev. Lett. 102, 136401 (2009).

[49] M. Eckstein and M. Kollar, Phys. Rev. B 78, 245113 (2008). 